\title{
APC, FBXW7, KRAS, PIK3CA, and TP53 Gene Mutations in Human Colorectal Cancer Tumors Frequently Detected by Next-Generation DNA Sequencing
}

Zheng Xu Cai ${ }^{1}, 2 \#$, Xu Dong Tang ${ }^{3 \#}$, He Li Gao ${ }^{1}$, Chuanning Tang ${ }^{4}$, Vijayalakshmi Nandakumar ${ }^{5}$, Lindsey Jones ${ }^{5}$, Hua Ye ${ }^{4}$, Feng Lou $^{4}$, Dandan Zhang ${ }^{4}$, Hong Sun ${ }^{4}$, Haichao Dong ${ }^{4}$, Guangchun Zhang ${ }^{4}$, Zhiyuan Liư ${ }^{4}$, Zhishou Dong ${ }^{4}$, Baishuai Guo ${ }^{4}, \mathrm{He} \mathrm{Yan}^{4}, \mathrm{Chaowei} \mathrm{Yan}^{4}$, Lu Wang ${ }^{4}$, Ziyi Su${ }^{4}$, Feng Yun Wang ${ }^{3}$, Jia Jia Wan ${ }^{1}$, Feng Qi Fang ${ }^{1}$, Hai Long Chen ${ }^{2}$, Dong Shang ${ }^{2}$, Xue F Huang ${ }^{5}$, Si-Yi Chen ${ }^{3,5^{*}}$ and Hui Shu Guo ${ }^{1,2^{*}}$

${ }^{1}$ Central laboratory, The First Affiliated Hospital of Dalian Medical University, Dalian 116011, China

${ }^{2}$ Institute of Integrative Medicine, Dalian Medical University, Dalian 116044, China

${ }^{3}$ Department of Gastroenterology, Xiyuan Hospital, China Academy of Chinese Medical Sciences, Beijing, 100091, China

${ }^{4}$ San Valley Biotechnology Inc., Beijing 100044, China

${ }^{5}$ Norris Comprehensive Cancer Center, Department of Molecular Microbiology and Immunology, Keck School of Medicine, University of Southern California, Los Angeles, CA 90033, USA

${ }^{\#}$ Both authors contributed equally to this study

*Corresponding authors: Hui-Shu Guo, The First Affiliated Hospital of Dalian Medical University, Dalian 116011, China, E-mail: guohuishu1@126.com

Si-Yi Chen, MD., Ph.D., Norris Comprehensive Cancer Center, Department of Molecular Microbiology and Immunology, Keck School of Medicine, University of Southern California, Los Angeles, CA 90033, USA, Tel: 323442 7727; Fax: 323442 7729; E-mail: siyichen@usc.edu

Received date: October 13, 2014, Accepted date: November 20, 2014, Published date: November 27, 2014

Copyright: ( 2014 Cai ZX, et al. This is an open-access article distributed under the terms of the Creative Commons Attribution License, which permits unrestricted use, distribution, and reproduction in any medium, provided the original author and source are credited.

\begin{abstract}
Colorectal cancer (CRC) is the fourth leading cause of cancer deaths worldwide. Genetic mutations have been linked to $5-10 \%$ of CRCs, with other environmental, genetic, and epigenetic factors influencing development and progression of the cancer. As individual cancers exhibit unique mutation patterns, identification of the distinctive CRC profile is essential to develop more effective target therapies. In this study, we used lon Torrent Ampliseq Cancer Panel to sequence 737 loci from 45 cancer-related genes to identify genetic mutations in 93 human colorectal cancer samples. Sequencing analysis revealed frequent missense mutations in APC (17.2\%), FBXW7 $(10.8 \%)$, KRAS $(50.5 \%)$, PIK3CA $(10.8 \%)$, and TP53 $(23.7 \%)$ in CRC samples of various histologic types, and additional mutations were also detected in other genes (BRAF, CTNNB1, NRAS, and SMAD4) at lesser frequencies. We also found common combination mutations between KRAS and TP53 (12.9\%), KRAS and APC (8.6\%), and KRAS and PIK3CA (8.6\%). Sequencing individual human cancers may be the key to developing more effective drugs to target individual, cancer-specific mutations. Identifying the complete mutation profile in CRCs for the application of personalized and tailored targeted therapy is critical for developing new cancer treatments. We believe a faster and cost effective genotyping tool such as lon Torrent sequencing technology will be greatly beneficial for the assignment of such specific therapeutics for CRCs in the near future.
\end{abstract}

Keywords: Colorectal cancer; Genetic mutations; Ion torrent sequencing; Targeted therapy; Personalized medicine

\section{Introduction}

Colorectal cancer (CRC) is the second most common malignancy in women and the third most common in men worldwide. In 2012, nearly 1.4 million new CRC cases were reported and roughly 694,000 associated deaths occurred worldwide, making it the fourth most common cause of death from cancer [1]. The same year, China alone accounted for more than 253,000 cases and nearly 140,000 CRC deaths. Nearly $60 \%$ of all CRC cases are reported from developed regions, and men have a significantly higher incidence and mortality rate than women [2]. While roughly one third of individuals diagnosed with CRC will die from the disease [3], an estimated $60 \%$ of CRC deaths could be prevented with routine screening of individuals over the age of 50 [4]. Despite continuing efforts to improve treatment options for colorectal cancer patients, the staggering incidence and high mortality rates prevail.

Like other cancers, a variety of environmental and genetic interactions are involved in the development of CRCs. Some major etiologic agents which influence sporadic CRC include inflammatory bowel disease (IBD), old age, male gender, high fat diet and obesity, tobacco and alcohol consumption, and sedentary lifestyle, while inherited genetics are responsible for between 5 and 10\% of CRC cases [5-8]. Some well characterized familial genetic syndromes have previously been identified which confer a higher risk of developing CRC: hereditary non-polyposis colorectal cancer (HNPCC), which is associated with mutated genes in the DNA mismatch repair pathway, including MSH2 and MLH1 [9,10]; and familial adenomatous polyposis (FAP), which is the consequence of germline mutations in the tumor suppressor gene APC. These APC mutations result in a non-functional APC protein product that fails to regulate the cellular overgrowth of polyps. Carriers of germline APC mutations have virtually $100 \%$ chance of developing CRC by age 40 , and most sporadic cancers have somatic mutations or deletions in this gene [11]. Loss of APC function is often followed by a series of other tumor suppressor gene mutations, including KRAS, PIK3CA, and TP53 [12]. In addition, epigenetic modifications like DNA methylation, histone modifications, and non-coding RNAs are particularly common in CRCs compared to other cancers [13]. The complex heterogeneity of CRCs highlight the need to identify biomarkers useful for target 
Citation: Cai ZX, Tang XD, Gao HL, Tang C, Nandakumar V, et al. (2014) APC, FBXW7, KRAS, PIK3CA, and TP53 Gene Mutations in Human Colorectal Cancer Tumors Frequently Detected by Next-Generation DNA Sequencing. J Mol Genet Med 8: 145. doi: $10.4172 / 1747-0862.1000145$

Page 2 of 11

therapies, as these genetic and epigenetic alterations have been demonstrated to correlate with prognosis and treatment responses [10]. For example, CRC tumors with KRAS mutations do not respond to commonly used drugs targeting EGFR, and chromosome instability found in more than $80 \%$ of CRCs is often associated with multi-drug resistance $[10,14]$.

Despite ongoing efforts to improve screening and treatment of colorectal cancers, further research is necessary to determine additional unknown genetic mutations and epigenetic influences which are involved in the development and progression of the disease. A variety of complex interactions between genetic and environmental factors contribute to the unique gene mutation profile exhibited in each individual tumor, and profiling an individual's cancer genome can reveal the oncogenic mechanisms that regulate the cancer. As such, there is accumulating evidence suggesting that individualized, tailored therapies are needed to more effectively treat cancer patients. Until recently, personalized medicine based on individual genome sequencing was expensive, time inefficient, and generally impractical; however, the recent development of semiconductor-based technology known as Ion Torrent sequencing, which differs from other sequencing methods in that it does not rely on modified nucleotides or optics, is making individual genome sequencing a possibility [15]. In this study, we have used Ion Torrent sequencing to analyze 93 clinical colorectal cancer samples to identify the genetic mutations in 737 loci from 45 known cancer-related genes.

\section{Materials and Methods}

\section{Ethics statement}

The study has been approved by the Human Research Ethics Committee of the First Affiliated Hospital of Dalian Medical University, China. The institutional ethics committee waived the need for consent for formalin-fixed, paraffin embedded (FFPE) tumor samples from the tumor tissue bank at the Department of Pathology of the hospital, for all samples and medical data used in this study have been irreversibly anonymized.

\section{Patient information}

All tumor samples used in the study were collected from the First Affiliated Hospital of Dalian Medical University, China. A total of 93 FFPE tumor samples from colorectal cancer patients were analyzed. Patients ranged from 29-86 years of age with a median age of 63 years (Table 1). The tumor samples were characterized by tissue origin (colon or rectum) and pathologic diagnosis (apophysis or ulcerative) (Table 2). Additional differentiation and TNM staging information is provided in Tables 3-5.

\begin{tabular}{|c|c|}
\hline Characteristic & All patients $(n=93)$ \\
\hline \multicolumn{2}{|l|}{ Age } \\
\hline Median (years) & 62 \\
\hline Range (years) & $29-86$ \\
\hline < 60 years: $\mathrm{n}(\%)$ & $36(38.7 \%)$ \\
\hline$\geq 60$ years: $\mathrm{n}(\%)$ & $57(61.3 \%)$ \\
\hline \multicolumn{2}{|l|}{ Gender } \\
\hline Male: n (\%) & $43(46.2 \%)$ \\
\hline Female: n (\%) & $50(53.8 \%)$ \\
\hline \multicolumn{2}{|l|}{ Pathologic Type } \\
\hline Apophysis: n (\%) & $18(19.4 \%)$ \\
\hline Ulcerative: n (\%) & $68(73.1 \%)$ \\
\hline Apophysis and Ulcerative: $\mathrm{n}(\%)$ & $1(1.1 \%)$ \\
\hline Unknown: n (\%) & $6(6.5 \%)$ \\
\hline \multicolumn{2}{|l|}{ Pathologic diagnosis location } \\
\hline Rectum: n (\%) & $81(87.1 \%)$ \\
\hline Colon: $\mathrm{n}(\%)$ & $11(11.8 \%)$ \\
\hline Rectum and Colon: $\mathrm{n}(\%)$ & $1(1.1 \%)$ \\
\hline
\end{tabular}

Table 1: Patients' characteristics for 93 human rectum and colon cancer samples. 
Citation: Cai ZX, Tang XD, Gao HL, Tang C, Nandakumar V, et al. (2014) APC, FBXW7, KRAS, PIK3CA, and TP53 Gene Mutations in Human Colorectal Cancer Tumors Frequently Detected by Next-Generation DNA Sequencing. J Mol Genet Med 8: 145. doi: $10.4172 / 1747-0862.1000145$

Page 3 of 11

\begin{tabular}{|c|c|c|c|c|c|c|c|c|c|c|}
\hline \multirow[b]{2}{*}{ Gene } & \multirow[b]{2}{*}{$\begin{array}{l}\text { Total } \\
\text { number of } \\
\text { samples } \\
\text { with } \\
\text { mutation (\% } \\
\text { in } 93 \\
\text { samples) }\end{array}$} & \multicolumn{2}{|l|}{ Sex } & \multicolumn{3}{|l|}{ Location } & \multicolumn{4}{|l|}{ Type } \\
\hline & & $\begin{array}{ll}\text { Male } & \\
\text { samples } \\
\text { with } \\
\text { mutation }(\% \\
\text { in } 4 \\
\text { samples) }\end{array}$ & $\begin{array}{l}\text { Female } \\
\text { samples } \\
\text { with } \\
\text { mutation } \\
\text { (\% in 50 } \\
\text { samples) }\end{array}$ & $\begin{array}{|ll|}\text { Colon } & \\
\text { samples } & \\
\text { with } & \\
\text { mutation } & (\% \\
\text { in } & 11 \\
\text { samples) } & \\
\end{array}$ & \begin{tabular}{ll|} 
Rectum & \\
samples & \\
with & \\
mutation & $(\%$ \\
in & 81 \\
samples) &
\end{tabular} & \begin{tabular}{|l|} 
Colon/ \\
Rectum \\
samples \\
with \\
mutation (\% \\
in \\
in
\end{tabular} & $\begin{array}{l}\text { Apophysis } \\
\text { samples with } \\
\text { mutation (\% in } \\
18 \text { samples) }\end{array}$ & $\begin{array}{l}\text { Ulcerative } \\
\text { samples } \\
\text { with } \\
\text { mutation } \\
(\% \text { in } 68 \\
\text { samples) }\end{array}$ & $\begin{array}{l}\text { Apophysis/ } \\
\text { Ulcerative } \\
\text { samples with } \\
\text { mutation (\% } \\
\text { in } 1 \text { sample) }\end{array}$ & $\begin{array}{l}\text { Unknown } \\
\text { samples } \\
\text { with } \\
\text { mutation } \\
\text { (\% in } 6 \\
\text { samples) }\end{array}$ \\
\hline APC & $16(17.2 \%)$ & $7(16.3 \%)$ & $9(18.0 \%)$ & $1(9.1 \%)$ & $15(18.5 \%)$ & $0(0.0 \%)$ & $2(11.1 \%)$ & $14(20.6 \%)$ & $0(0.0 \%)$ & $0(0.0 \%)$ \\
\hline BRAF & $1(1.1 \%)$ & $0(0.0 \%)$ & $1(2.0 \%)$ & $0(0.0 \%)$ & $1(1.2 \%)$ & $0(0.0 \%)$ & $0(0.0 \%)$ & $1(1.5 \%)$ & $0(0.0 \%)$ & $0(0.0 \%)$ \\
\hline CTNNB1 & $1(1.1 \%)$ & $0(0.0 \%)$ & $1(2.0 \%)$ & $0(0.0 \%)$ & $1(1.2 \%)$ & $0(0.0 \%)$ & $0(0.0 \%)$ & $1(1.5 \%)$ & $0(0.0 \%)$ & $0(0.0 \%)$ \\
\hline FBXW7 & $10(10.8 \%)$ & $7(16.3 \%)$ & $3(6.0 \%)$ & $2(18.2 \%)$ & $8(9.9 \%)$ & $0(0.0 \%)$ & $3(16.7 \%)$ & $7(10.3 \%)$ & $0(0.0 \%)$ & $0(0.0 \%)$ \\
\hline KRAS & 47 (50.5\%) & $22(51.2 \%)$ & $25(50.0 \%)$ & $6(54.5 \%)$ & $40(49.4 \%)$ & $1(100.0 \%)$ & $9(50.0 \%)$ & $33(48.5 \%)$ & $1(100.0 \%)$ & $4(66.7 \%)$ \\
\hline NRAS & $3(3.2 \%)$ & $1(2.3 \%)$ & $2(4.0 \%)$ & $0(0.0 \%)$ & $3(3.7 \%)$ & $0(0.0 \%)$ & $1(5.6 \%)$ & $2(2.9 \%)$ & $0(0.0 \%)$ & $0(0.0 \%)$ \\
\hline PIK3CA & $10(10.8 \%)$ & $7(16.3 \%)$ & $3(6.0 \%)$ & $1(9.1 \%)$ & $8(9.9 \%)$ & $1(100.0 \%)$ & $3(16.7 \%)$ & $6(8.8 \%)$ & $1(100.0 \%)$ & $0(0.0 \%)$ \\
\hline SMAD4 & $2(2.2 \%)$ & $1(2.3 \%)$ & $1(2.0 \%)$ & $0(0.0 \%)$ & $2(2.5 \%)$ & $0(0.0 \%)$ & $1(5.6 \%)$ & $1(1.5 \%)$ & $0(0.0 \%)$ & $0(0.0 \%)$ \\
\hline TP53 & $22(23.7 \%)$ & $8(18.6 \%)$ & $14(28.0 \%)$ & $3(27.3 \%)$ & $18(22.2 \%)$ & $1(100.0 \%)$ & $7(38.9 \%)$ & $13(19.1 \%)$ & $1(100.0 \%)$ & $1(16.7 \%)$ \\
\hline
\end{tabular}

Table 2: Detected mutations frequencies of 93 human rectum and colon cancers based on sex, diagnostic location (colon vs. rectum), and type (apophysis vs. ulcerative).

\section{DNA preparation}

Formalin-fixed, paraffin embedded (FFPE) tissue samples were deparaffinized in xylene prior to extraction of $3-5 \mu \mathrm{m}$ thick sections. The QIAamp DNA Mini Kit (Qiagen) was then used to isolate DNA as per the manufacturer's instructions.

\section{Ion torrent PGM library preparation and sequencing}

An Ion Torrent adapter-ligated library was constructed with the Ion AmpliSeq Library Kit 2.0 (Life Technologies, Part \#4475345 Rev. A) following the manufacturer's protocol, and as in our previous publications [16,17]. The Personalized Cancer Mutation Panel used for this study targets 737 loci to detect mutations in the following 45 cancer-related genes: ABL1, AKT1, ALK, APC, ATM, BRAF, CDH1, CDKN2A, CSF1R, CTNNB1, EGFR, ERBB2, ERBB4, FBXW7, FGFR1, FGFR2, FGFR3, FLT3, GNAS, HNF1A, HRAS, IDH1, JAK3, KDR, KIT, KRAS, MET, MLH1, MPL, NOTCH1, NPM1, NRAS, PDGFRA, PIK3CA, PTEN, PTPN11, RB1, RET, SMAD4, SMARCB1, SMO, SRC, STK11, TP53, and VHL.

\section{Sequence coverage}

For the 93 CRC samples analyzed, the mean read length was 76 bp and the average sequence per sample was approximate $18 \mathrm{Mb}$. With normalization to 300,000 reads per specimen, there was an average of 1,639 reads per amplicon (range: 15 to 3,979) (Figure 1A); 176/189 (93.1\%) amplicons averaged at least 100 reads, and 165/189 (87.3\%) amplicons averaged at least 300 reads (Figure 1B).

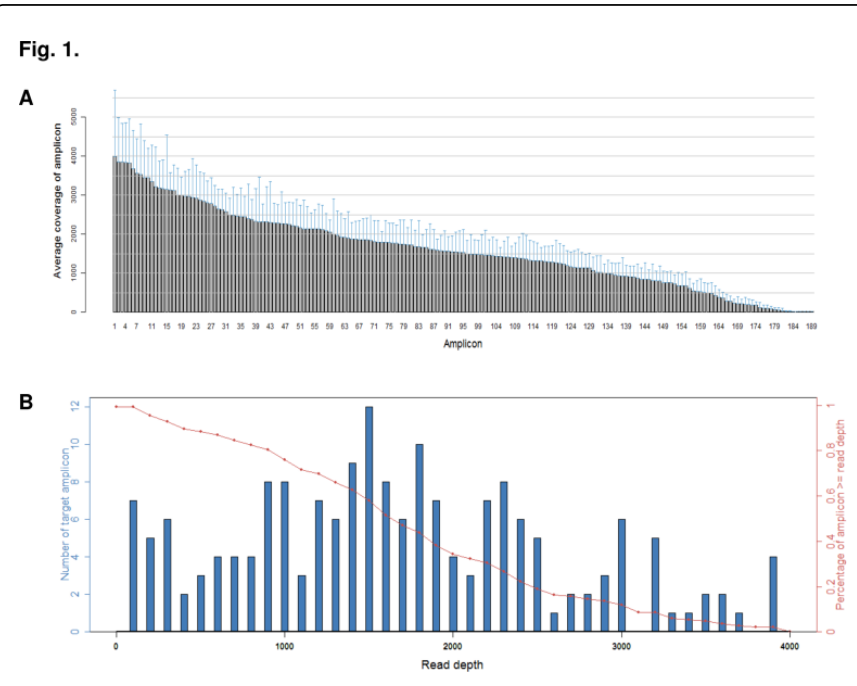

Figure 1: Sequence read distribution across 189 amplicons generated from 93 specimens, normalized to 300,000 reads per sample

Average number of reads observed for each amplicon.

Number of targets with a given read depth, sorted in bins of 100 reads. (blue bar means Number of targets within read depth, red line means $\%$ of targets $\geq$ read depth)

\section{Variant calling}

The Ion Torrent platform-specific pipeline software Torrent Suite initially processed data from the PGM runs to generate sequence 
Citation: Cai ZX, Tang XD, Gao HL, Tang C, Nandakumar V, et al. (2014) APC, FBXW7, KRAS, PIK3CA, and TP53 Gene Mutations in Human Colorectal Cancer Tumors Frequently Detected by Next-Generation DNA Sequencing. J Mol Genet Med 8: 145. doi: $10.4172 / 1747-0862.1000145$

Page 4 of 11

reads, trim adapter sequences, filter, and remove any poor signalprofile reads. Initial variant calling from the Ion AmpliSeq sequencing data was performed with Torrent Suite Software v3.0 with a plug-in "variant caller" program. With the aim of eliminating erroneous base calling, several filtering steps were utilized to produce final variant calling, including defining variant frequency and coverage depth, eliminating DNA strand-specific errors, defining variants within 727 hotspots, and removing variants in amplicon AMPL339432 (PIK3CA, exon13, chr3:178938822-178938906), and as further detailed in our previous publications [16,17]. Additionally, as the JAK2 gene locus generated false deletion data, sequencing data from this locus was excluded from further analysis. Furthermore, Sanger's sequencing was frequently used to confirm the detected missense mutations.

\section{Somatic mutations}

Detected mutations were compared to variants in the 1000 Genomes Project [18] and 6,500 exomes of the National Heart, Lung, and Blood Institute Exome Sequencing Project [19] to distinguish somatic mutations and germline mutations.

\section{Bioinformatical and experimental validation}

We used the COSMIC (version 64) [20], My Cancer Genome database (http://www.mycancergenome.org/), and additional publications to evaluate reappearing mutations in CRCs (Supplemental Table 1). Additionally, some of the detected mutations were confirmed by Sanger's sequencing (Supplemental Table 2).

\section{Results and Discussion}

\section{Mutation analysis of human colorectal cancers with ion ampliseq cancer panel}

A total of 93 human colon and rectal cancer samples were analyzed using Ion Torrent Ampliseq Cancer Panel to identify mutations in 737 loci from 45 oncogenes and tumor suppressor genes. The sequenced data were processed and we found frequent mutations in the following genes: APC (17.2\%), FBXW7 (11.8\%), KRAS (51.6\%), PIK3CA (10.8\%), and TP53 (23.7\%). Mutations were also identified in the following genes at lesser frequencies: BRAF (1.1\%), CTNNB1 (1.1\%), NRAS (3.2\%), and SMAD4 (2.2\%). When sufficient DNA was available, Sanger's sequencing was used to confirm these mutations, and for those samples, Sanger's sequencing confirmed $100 \%$ of these mutations (40/40) (Supplemental Table 2) (Supplementary Figure $1-40)$.

Overall, mutations were found in 70 of the 93 samples $(75.3 \%)$. Specifically, 38 (40.9\%) samples contained one mutation (Table 3), 20 $(21.5 \%)$ had two mutations (Table 4 ), and 12 (12.9\%) had three mutations (Table 5) among the 45 genes screened. The number of mutations found per sample tends to correlate with staging of the cancer: for samples with one mutation and reported staging, 30\% were stage 3; for samples with two mutations and reported staging, $66.7 \%$ were stage 3 or higher; and for samples with three mutations and reported staging, $77.8 \%$ were stage 3 or higher. $81.8 \%$ of colon samples had at least one mutation, whereas $74.1 \%$ of rectal samples had at least one mutation. Proportionally, the APC mutation rate was twice as high in rectal samples vs. those from the colon, which is consistent with CRC COSMIC data [21], whereas the opposite was true for FBXW7 mutations. The other mutations were found at roughly equal proportions between pathological locations. Mutations were also found to be proportionally similar between males and females, with the exception of FBXW7 (18.6\% vs. $6.0 \%$, respectively), PIK3CA $(16.3 \%$ vs. $6.0 \%$, respectively), and TP53 (18.6\% vs. $28.0 \%$, respectively) (Table 2 ).

\begin{tabular}{|c|c|c|c|c|c|c|c|}
\hline Gene & Mutation & Sex & Age & Location & Type & Differentiation & TNM Stage \\
\hline APC & Q1303* & $\mathrm{F}$ & 57 & Rectum & Ulcerative & Middle & ND \\
\hline APC & E1306* & $\mathrm{F}$ & 64 & Rectum & Ulcerative & Middle-Low & ND \\
\hline APC & Q1367* & M & 58 & Rectum & Ulcerative & Middle-High & ND \\
\hline APC & $\mathrm{R} 1450^{*}$ & M & 64 & Colon & Ulcerative & Middle-Low & ND \\
\hline BRAF & V600E & $\mathrm{F}$ & 79 & Rectum & Ulcerative & Middle & ND \\
\hline CTNNB1 & S45F & $\mathrm{F}$ & 56 & Rectum & Ulcerative & Middle & 1 \\
\hline FBXW7 & R505C & $\mathrm{F}$ & 57 & Rectum & Ulcerative & Middle & ND \\
\hline KRAS & $\mathrm{G} 12 \mathrm{C}$ & M & 59 & Rectum & Unknown & Middle-High & II \\
\hline KRAS & $\mathrm{G} 12 \mathrm{D}$ & $M$ & 54 & Colon & Unknown & Middle & ND \\
\hline KRAS & G12D & $\mathrm{F}$ & 68 & Rectum & Ulcerative & Middle & ND \\
\hline KRAS & G12D & $\mathrm{F}$ & 42 & Rectum & Unknown & Middle & 1 \\
\hline KRAS & G12D & $M$ & 68 & Rectum & Ulcerative & Middle & III \\
\hline KRAS & $\mathrm{G} 12 \mathrm{D}$ & $\mathrm{F}$ & 60 & Rectum & Apophysis & Middle & ND \\
\hline
\end{tabular}


Citation: Cai ZX, Tang XD, Gao HL, Tang C, Nandakumar V, et al. (2014) APC, FBXW7, KRAS, PIK3CA, and TP53 Gene Mutations in Human Colorectal Cancer Tumors Frequently Detected by Next-Generation DNA Sequencing. J Mol Genet Med 8: 145. doi: $10.4172 / 1747-0862.1000145$

Page 5 of 11

\begin{tabular}{|c|c|c|c|c|c|c|c|}
\hline KRAS & G12D & M & 82 & Rectum & Ulcerative & Middle & 1 \\
\hline KRAS & G12D & $\mathrm{F}$ & 78 & Rectum & Ulcerative & Middle & ND \\
\hline KRAS & G12D & $M$ & 58 & Rectum & Ulcerative & Middle-High & III \\
\hline KRAS & G12D & $\mathrm{F}$ & 63 & Rectum & Ulcerative & High & II \\
\hline KRAS & G12S & $\mathrm{F}$ & 72 & Colon & Ulcerative & Middle & III \\
\hline KRAS & G12V & M & 49 & Rectum & Ulcerative & Middle-High & II \\
\hline KRAS & G12V & $\mathrm{F}$ & 67 & Rectum & Ulcerative & Unknown & ND \\
\hline KRAS & G13D & $F$ & 75 & Rectum & Ulcerative & Low & II \\
\hline KRAS & G13D & $\mathrm{F}$ & 58 & Rectum & Ulcerative & Middle & ND \\
\hline KRAS & G13D & $\mathrm{F}$ & 70 & Rectum & Ulcerative & Middle & II \\
\hline KRAS & G13D & M & 62 & Rectum & Apophysis & Middle & II \\
\hline KRAS & G13D & $\mathrm{F}$ & 53 & Rectum & Ulcerative & Middle-High & III \\
\hline KRAS & G13D & M & 86 & Colon & Ulcerative & Middle-High & II \\
\hline KRAS & G13D & $F$ & 71 & Rectum & Unknown & High & III \\
\hline KRAS & G13D & $\mathrm{F}$ & 49 & Rectum & Ulcerative & Unknown & ND \\
\hline KRAS & A146T & $M$ & 60 & Rectum & Ulcerative & Middle & ND \\
\hline NRAS & Q61K & $\mathrm{F}$ & 67 & Rectum & Apophysis & Middle & I \\
\hline NRAS & Q61R & M & 49 & Rectum & Ulcerative & Middle & III \\
\hline PIK3CA & E545K & M & 50 & Colon & Ulcerative & Middle-High & ND \\
\hline TP53 & Y163C & $\mathrm{F}$ & 59 & Rectum & Apophysis & Middle & ND \\
\hline TP53 & $\mathrm{R} 175 \mathrm{H}$ & $\mathrm{F}$ & 67 & Colon & Apophysis & Middle-High & II \\
\hline TP53 & $\mathrm{R} 175 \mathrm{H}$ & M & 62 & Rectum & Ulcerative & Middle-High & ND \\
\hline TP53 & $\mathrm{C} 176 \mathrm{~F}$ & $F$ & 72 & Rectum & Unknown & Middle & ND \\
\hline TP53 & Y220C & $\mathrm{F}$ & 60 & Rectum & Ulcerative & Middle & II \\
\hline TP53 & $\mathrm{C} 275 \mathrm{Y}$ & M & 64 & Rectum & Apophysis & Middle & 1 \\
\hline
\end{tabular}

Table 3: Single point mutations detected among 93 colorectal cancer samples.

${ }^{*}$ Nonsense mutations resulting in STOP codon; ND: Not determined

\begin{tabular}{|c|c|c|c|c|c|c|c|c|c|}
\hline Gene 1 & Mutation 1 & Gene 2 & Mutation 2 & Sex & Age & Location & Type & Differentiation & $\begin{array}{l}\text { TNM } \\
\text { Stage }\end{array}$ \\
\hline KRAS & G12D & APC & $\mathrm{R} 876^{*}$ & $M$ & 79 & Rectum & Ulcerative & Middle & ND \\
\hline KRAS & G12D & APC & $\mathrm{R} 876^{*}$ & $\mathrm{~F}$ & 79 & Rectum & Ulcerative & Middle & II \\
\hline KRAS & G12D & APC & $\mathrm{E} 1309 \mathrm{fs}{ }^{*} 4$ & $\mathrm{~F}$ & UNK & Rectum & Ulcerative & High & II \\
\hline KRAS & G13D & APC & Q1378* & $M$ & 70 & Rectum & Ulcerative & Middle-High & III \\
\hline KRAS & G13D & APC & $\mathrm{R} 1450^{*}$ & $\mathrm{~F}$ & 76 & Rectum & Apophysis & Middle & ND \\
\hline KRAS & G12D & TP53 & $\mathrm{R} 158 \mathrm{H}$ & $\mathrm{F}$ & 81 & Rectum & Ulcerative & Middle & III \\
\hline
\end{tabular}


Citation: Cai ZX, Tang XD, Gao HL, Tang C, Nandakumar V, et al. (2014) APC, FBXW7, KRAS, PIK3CA, and TP53 Gene Mutations in Human Colorectal Cancer Tumors Frequently Detected by Next-Generation DNA Sequencing. J Mol Genet Med 8: 145. doi:

Page 6 of 11

\begin{tabular}{|l|l|l|l|l|l|l|l|l|l|}
\hline KRAS & G12D & TP53 & Y220C & F & 45 & Rectum & Ulcerative & Middle \\
\hline KRAS & G12V & TP53 & F270S & M & 66 & Colon & Apophysis & Middle-Low \\
\hline KRAS & G13D & TP53 & V173L & F & 58 & Rectum & Ulcerative & Middle-Low \\
\hline KRAS & G13D & TP53 & R248W & F & 49 & Rectum & Ulcerative & Middle & III \\
\hline KRAS & G12D & PIK3CA & E545K & F & 57 & Rectum & Apophysis & Middle-Low \\
\hline KRAS & G12V & PIK3CA & E545K & M & 69 & Rectum & Ulcerative & Middle & III \\
\hline KRAS & G12V & PIK3CA & E545K & M & 61 & Rectum & Ulcerative & Low & ND \\
\hline KRAS & G12C & FBXW7 & R465C & M & 63 & Colon & Ulcerative & Middle & ND \\
\hline TP53 & R273C & APC & Q1367 & M & 63 & Rectum & Ulcerative & Middle & ND \\
\hline TP53 & E285K & APC & Q1367 & F & 53 & Rectum & Apophysis & Middle & III \\
\hline TP53 & A159V & NRAS & G13R & F & 58 & Rectum & Ulcerative & Middle & III \\
\hline TP53 & G245V & SMAD4 & Q245* & F & 65 & Rectum & Ulcerative & Middle & III \\
\hline FBXW7 & R465H & APC & K1370 & F & 51 & Rectum & Ulcerative & Middle-Low & ND \\
\hline FBXW7 & R465C & PIK3CA & H1047R & M & 79 & Rectum & Ulcerative & Middle & III \\
\hline
\end{tabular}

Table 4: Combination point mutations in two genes detected among 93 colorectal cancer samples.

${ }^{*}$ Nonsense mutations resulting in STOP codon; fs: Frameshift mutation; ND: Not determined

\begin{tabular}{|l|l|l|l|l|l|l|l|l|l|l|l|}
\hline Gene 1 & Mutation 1 & Gene 2 & Mutation 2 & Gene 3 & Mutation 3 & Sex & Age & Location & Type & Differentiation & $\begin{array}{l}\text { TNM } \\
\text { Stage }\end{array}$ \\
\hline KRAS & G12D & TP53 & R175H & APC & Q1303* & F & 76 & Rectum & Ulcerative & Middle & III \\
\hline KRAS & G12D & TP53 & R273C & APC & E1309fs 4 & F & 75 & Rectum & Ulcerative & Middle & II \\
\hline KRAS & G12V & TP53 & R248W & FBXW7 & R465C & M & 59 & Rectum & Apophysis & Middle & II \\
\hline KRAS & A146T & TP53 & P152L & FBXW7 & R505L & M & 80 & Colon & Ulcerative & Middle & III \\
\hline KRAS & G12D & TP53 & R213* & PIK3CA & E545K & M & 56 & Rectum & Apophysis & Middle & ND \\
\hline KRAS & G12D & TP53 & E298* & PIK3CA & E542K & M & 67 & Both & Both & Unknown & III \\
\hline KRAS & G13D & TP53 & R175H & PIK3CA & M1043V & F & 29 & Rectum & Ulcerative & Mid-High & III \\
\hline KRAS & G12D & PIK3CA & E545K & FBXW7 & R278* & F & 67 & Rectum & Apophysis & Middle & III \\
\hline KRAS & G12S & PIK3CA & E545K & FBXW7 & R479Q & M & 73 & Rectum & Ulcerative & Middle & III \\
\hline KRAS & G13A & KRAS & G13R & APC & R1114* & M & 77 & Rectum & Ulcerative & Middle & ND \\
\hline KRAS & G12D & SMAD4 & R361H & FBXW7 & R465H & M & 57 & Rectum & Apophysis & Middle & ND \\
\hline FBXW7 & R278* & FBXW7 & R465H & APC & K1370* & M & 67 & Rectum & Ulcerative & Middle & IV \\
\hline
\end{tabular}

Table 5: Combination point mutations in three genes detected among 93 colorectal cancer samples.

${ }^{*}$ Nonsense mutations resulting in STOP codon; fs: Frameshift mutation; ND: Not determined

\section{APC mutations}

Of the 93 sequenced colorectal cancer samples, 16 APC mutations were found, all of which were located in exon 15 (Figure 2A). Fourteen of the 16 mutations detected resulted in a stop codon (R876 ${ }^{\star}, \mathrm{R} 1114^{*}$, Q1303*, E1306, Q1367*, Q1378*, R1450*) and truncated protein product, and the other two mutations were deletions resulting in a frameshift (E1309fs $\left.{ }^{*} 4\right)$ (Figure 2B and 2C). The APC gene consists of 21 exons and encodes a 2,843 amino acid protein [22,23]. Exon 15 comprises $77 \%$ of the coding sequence of APC and is the most 
Citation: Cai ZX, Tang XD, Gao HL, Tang C, Nandakumar V, et al. (2014) APC, FBXW7, KRAS, PIK3CA, and TP53 Gene Mutations in Human Colorectal Cancer Tumors Frequently Detected by Next-Generation DNA Sequencing. J Mol Genet Med 8: 145. doi: $10.4172 / 1747-0862.1000145$

Page 7 of 11

common target for both germline and somatic mutations [24]. Specifically, a mutation cluster region (MCR) in exon 15 between codons 1,286 and 1,513, which comprises only $10 \%$ of the total APC coding sequence, accounts for greater than $90 \%$ of APC mutations in all CRCs [25].

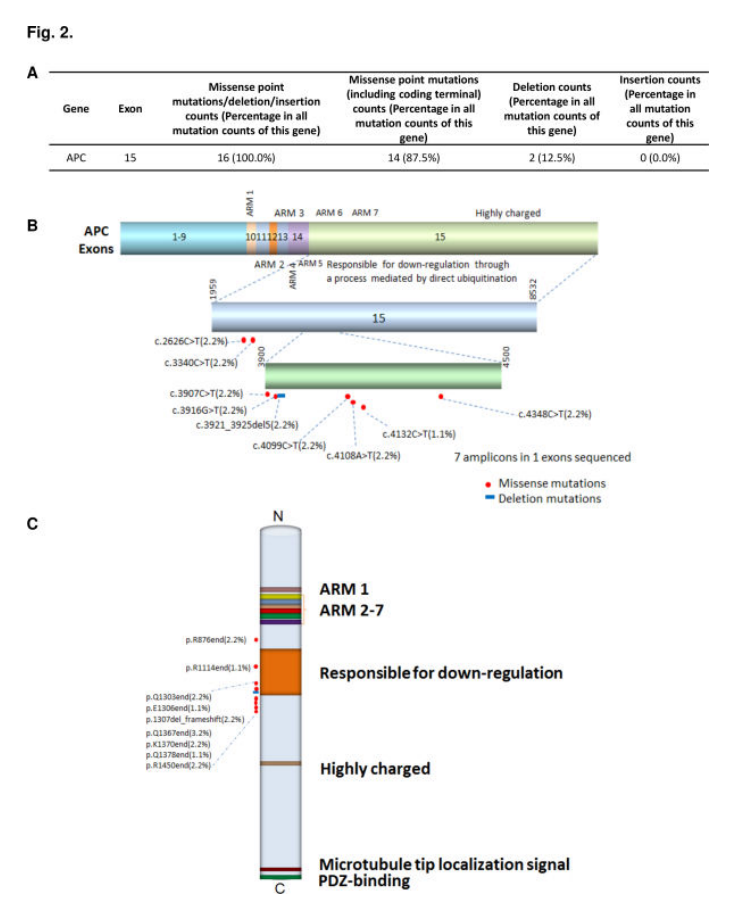

Figure 2: Missense mutation distribution in the exons and functional domains of APC.

Frequencies of detected mutations in different exons.

Mutation distribution in exons.

Mutation distribution in functional domains.

APC is an important tumor suppressor known to play a role in colorectal cancer. Mutations in the APC gene leads to a condition known as familial adenomatous polyposis (FAP), in which the intestinal epithelium is studded with hundreds to thousands of benign polyps, some of which may progress to invasive cancers if left untreated [26]. The vast majority of FAP-associated APC mutations lead to a truncated protein. The protein translated from the APC gene product interacts with cytoplasmic beta-catenin, mediates its degradation through ubiquitination, and thereby downregulates transcription exerted by the $\beta$-catenin-Tcf complex [24]. However, in the absence of a functional APC protein, $\beta$-catenin is stabilized and accumulates in the cytoplasm [24]. This results in constitutive transcriptional activation of Tcf-responsive genes, which may contribute to cancer progression [24].

\section{FBXW7 mutations}

Eleven FBXW7 mutations were identified in ten samples (10.8\%), which is consistent with previous findings that roughly $10 \%$ of CRCs have FBXW7 mutations [27].
These mutations were distributed along the following exons: R278* in exon $2(18.2 \%), \mathrm{R} 465 \mathrm{C} / \mathrm{H}$ in exon $6(54.5 \%)$, and R479Q and R505C/L in exon 9 (27.3\%) (Figure 3).

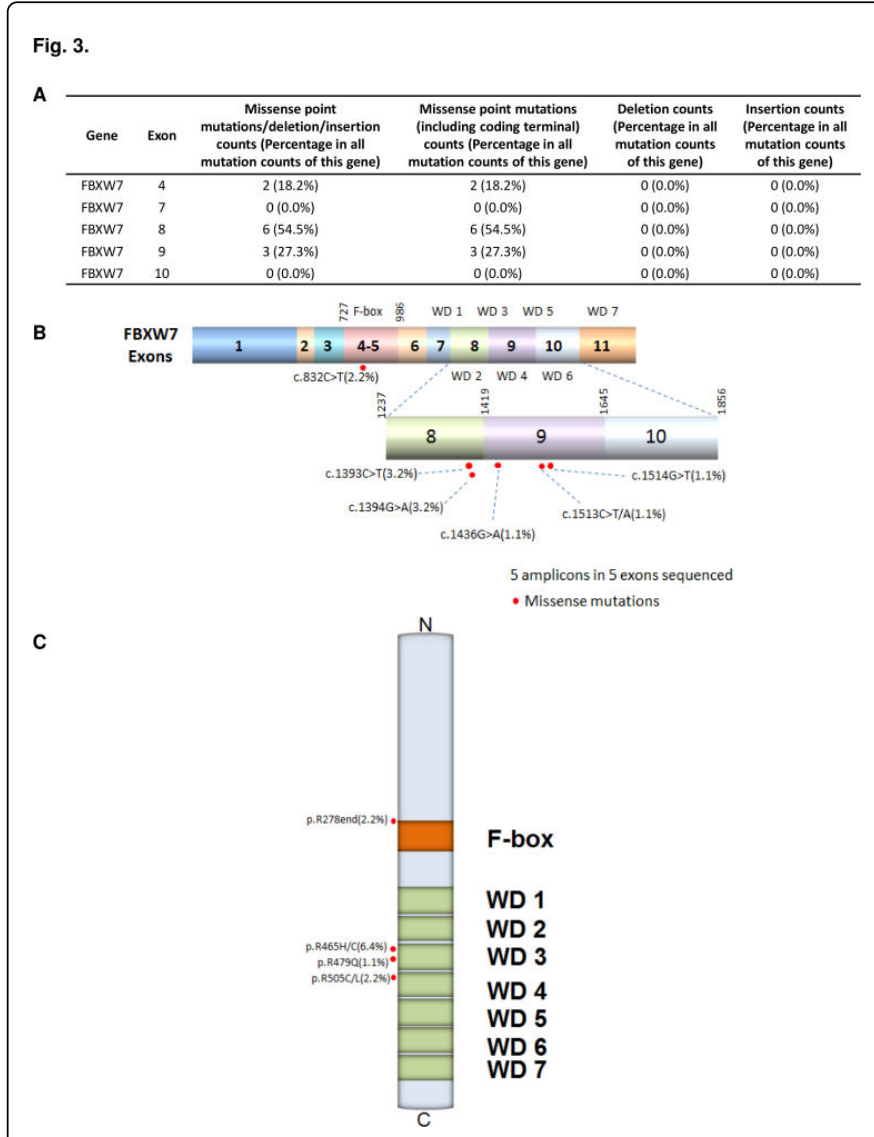

Figure 3: Missense mutation distribution in the exons and functional domains of FBXW7.

Frequencies of detected mutations in different exons.

Mutation distribution in exons.

Mutation distribution in functional domains.

FBXW7 (Cdc4) is a potential tumor suppressor that regulates ubiquitination and proteolysis of multiple targets. It negatively regulates multiple proteins with established roles in the control of cell division and growth, including cyclin E, c-Jun, c-Myc, mTOR, and Notch. FBXW7 proteins contain domains called WD4 repeats, which can form eight-bladed, barrel-shaped $\beta$-propeller-like binding pockets for substrates $[28,29]$. Arginine residues at the top of these propellerlike pockets directly interact with the recognition sequences in substrate $[28,29]$, and mutations at codons 465,479 , and 505 lie at the $\beta$-propeller tips which interact with FBXW7 substrates [30]. Mutations in FBXW7 impair Cyclin E degradation and are associated with decreased genetic stability and impaired growth regulation, contributing to the progression of CRC $[31,32] 32$.

\section{KRAS mutations}

KRAS mutations have been reported in up to $50 \%$ of all human CRCs [33]. In accordance, 47 of the samples in our study (50.5\%) 
Citation: Cai ZX, Tang XD, Gao HL, Tang C, Nandakumar V, et al. (2014) APC, FBXW7, KRAS, PIK3CA, and TP53 Gene Mutations in Human Colorectal Cancer Tumors Frequently Detected by Next-Generation DNA Sequencing. J Mol Genet Med 8: 145. doi: $10.4172 / 1747-0862.1000145$

Page 8 of 11

contained at least one KRAS mutation affecting the GTP binding domains, where $90.2 \%$ of these mutations were missense in exon 2 (G12C/D/S/V and $\mathrm{G} 13 \mathrm{~A} / \mathrm{D} / \mathrm{R})$, and $4.2 \%$ were found in exon 4 (A146T) (Figure 4). Consistent with our results, roughly $90 \%$ of all KRAS mutations in human cancers occur in codon 12 and 13, whereas the A146 mutation occurs in approximately $4 \%$ of CRCs [34]. These mutations are thought to occur early in carcinogenesis between the transitions from benign to late stage adenomas [34].Various CRC studies suggest that KRAS mutations in codons 12 and 13 are generally predictive of a poorer prognosis [35], whereas mutations in exon 4 are associated with a more favorable clinical outcome in CRC patients [36].

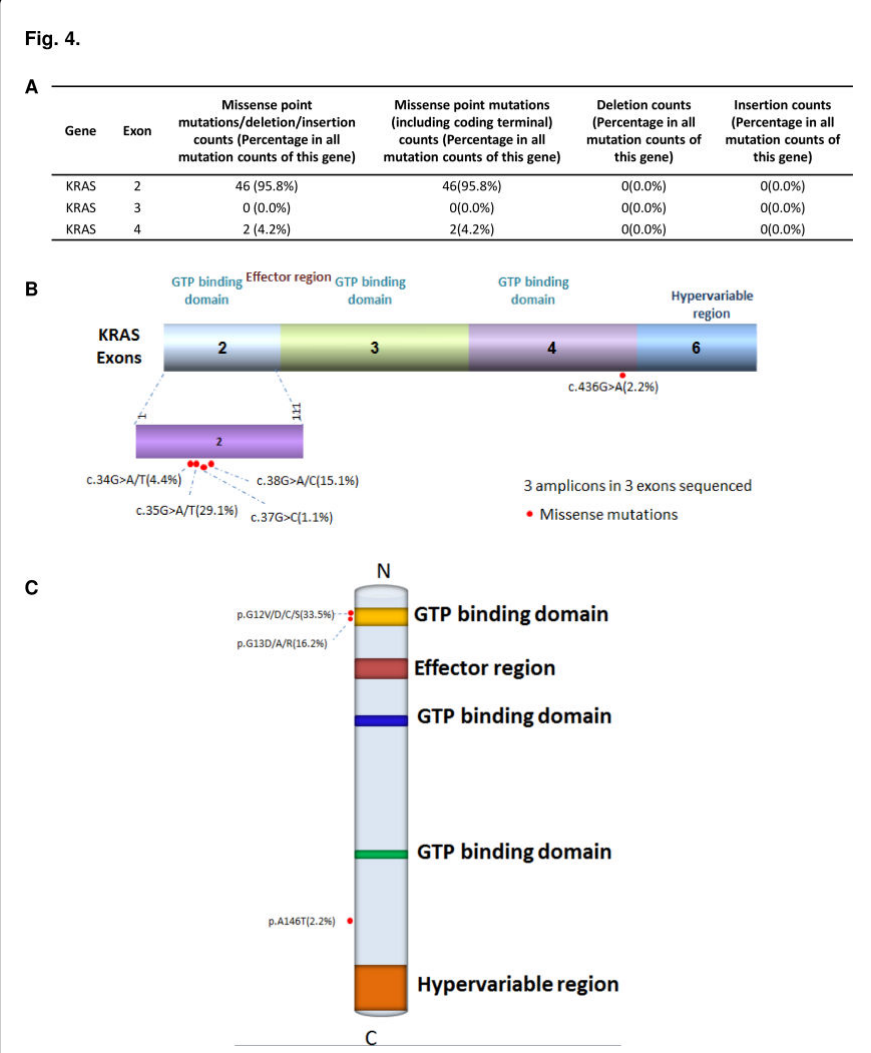

Figure 4: Missense mutation distribution in the exons and functional domains of KRAS.

Frequencies of detected mutations in different exons.

Mutation distribution in exons.

Mutation distribution in functional domains.

KRAS is an oncogene involved in the MAPK signaling cascade, and KRAS mutations have been shown to promote adenoma growth in the progression of CRC development. KRAS binds to GTP in the active state and possesses an intrinsic enzymatic activity which cleaves the terminal phosphate of the nucleotide and converts it to GDP. Upon conversion of GTP to GDP, KRAS is turned off [37], and the result of KRAS mutations is constitutive activation of KRAS signaling pathways. Once turned on, KRAS recruits and activates proteins necessary for the propagation of growth factor and other receptors' signal, such as c-Raf and PI3-kinase [37]. Thus early KRAS mutations influence the signal transduction process which promotes intestinal adenomatous growth [33].

\section{PIK3CA mutations}

An estimated $10-30 \%$ of all CRCs have PIK3CA mutations [38]. Accordingly, ten of our 93 CRC samples (10.8\%) harbored missense mutations in the PIK3CA gene (Figure 5A), where $80.0 \%$ were found in exon 9 (E542K and E545K) and 20.0\% in exon 20 (M1043V and H1047R). These exons encode for the helical and kinase domain of PI3K, respectively (Figure 5B and 5C), and roughly $80 \%$ of oncogenic PIK3CA mutations are located in these mutation hotspots [39].

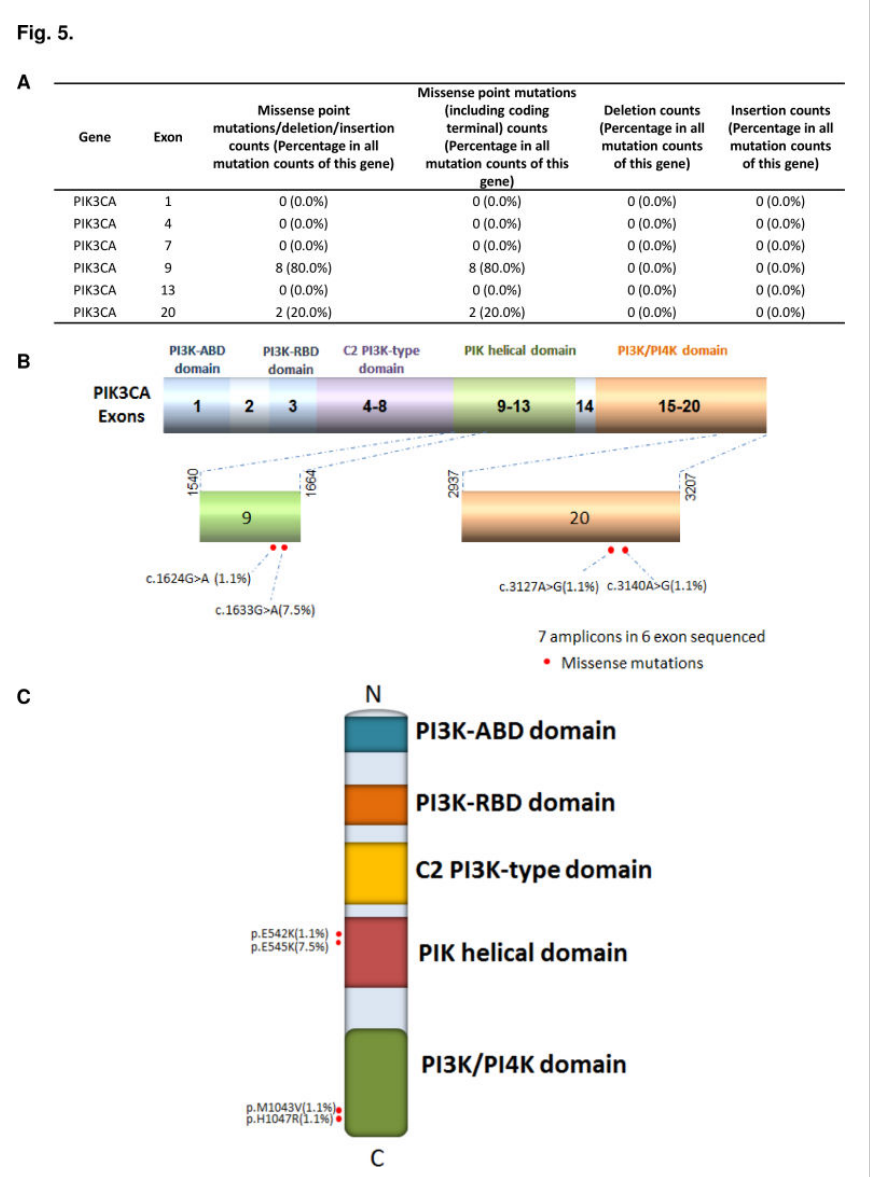

Figure 5: Missense mutation distribution in the exons and functional domains of PIK3CA.

Frequencies of detected mutations in different exons.

Mutation distribution in exons.

Mutation distribution in functional domains.

PIK3CA (phosphatidylinositol-4,5-bisphosphate 3-kinase, catalytic subunit alpha) is a lipid kinase capable of phosphorylating the $3^{\prime} \mathrm{OH}$ of the inositol ring of phosphoinositides. PI3K is responsible for coordinating a diverse range of cellular functions including proliferation, migration, and cell survival, as well as oncogenic transformations. Mutations in the kinase and helical domains of PI3K is associated with increased lipid kinase activity, and activation of 
Citation: Cai ZX, Tang XD, Gao HL, Tang C, Nandakumar V, et al. (2014) APC, FBXW7, KRAS, PIK3CA, and TP53 Gene Mutations in Human Colorectal Cancer Tumors Frequently Detected by Next-Generation DNA Sequencing. J Mol Genet Med 8: 145. doi: $10.4172 / 1747-0862.1000145$

Page 9 of 11

downstream Akt signaling caused by these mutations interferes with other signaling pathways that contribute to oncogenicity [40-42].

\section{TP53 mutations}

TP53 incurred several mutations across multiple exons in our sample set: 45.5\% in exon 5 (P152L, R158H, A159V, Y163C, V173L/G, $\mathrm{R} 175 \mathrm{H}$, and $\mathrm{C} 176 \mathrm{~F}), 13.6 \%$ in exon 6 (R213 ${ }^{*}$ and $\left.\mathrm{Y} 220 \mathrm{C}\right), 13.6 \%$ in exon 7 (G245V and R248W), and 27.3\% in exon 8 (F270S, R273C, C275Y, C277F, E285K, and E298*) (Figure 6). Most of these mutations are at known hotspot mutation sites, with the exception of A159V, F270S, C277F, E285K, and E298*, which are common mutations, and V173G, which is not common. All of these mutations occurred in the DNA binding region, and some of these occurred in the L2-L3 region (V173L/G, R175H, C176F, G245V, and R248W). Consistent with our findings, most TP53 mutations cluster in the TP53 DNA-binding domain, which encompasses exons 5 through 8 and spans approximately 180 codons [43]. Our sample set had a lower TP53 mutation frequency than expected $(23.7 \%)$, as previous studies have found TP53 mutations in $50-70 \%$ of colorectal cancers [44], which may reflect differences in populations or our limited sample size.

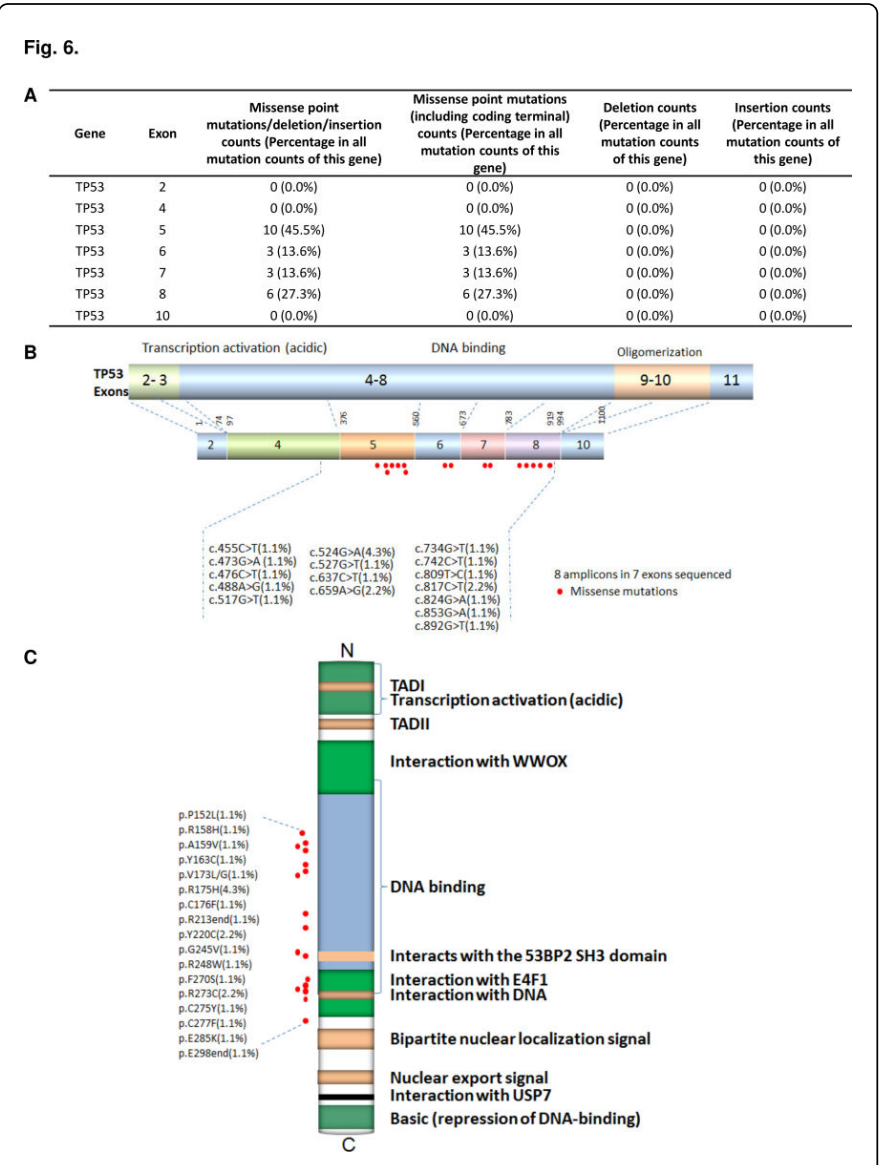

Figure 6: Missense mutation distribution in the exons and functional domains of TP53.

Frequencies of detected mutations in different exons.

Mutation distribution in exons.

Mutation distribution in functional domains.
Abnormality of the TP53 gene is one of the most common events in CRCs and plays an important role in the tumorigenesis of colorectal epithelial cells. TP53 mutations are thought to be relatively late events in CRC development, with a loss of TP53-mediated apoptotic pathways as an important factor in the progression from an adenoma to a malignant tumor [25]. Most TP53 missense mutations lead to the synthesis of a stable protein, but this lacks specific DNA-binding and transactivation functions and accumulates in the nucleus of cells. Such mutant proteins become inactive and lose the ability to transactivate downstream target genes that regulate cell cycle and apoptosis [45]. Apart from these mutations affecting the role of TP53 as a tumorsuppressor protein, TP53 mutations also endow the mutant protein with 'gain-of-function' (GOF) activities, which can contribute actively to various stages of tumor progression, including distant metastases and an increased resistance to anticancer treatments $[46,47]$.

\section{Combination mutations}

Roughly one third of the CRC samples $(34.4 \%)$ contained more than one mutation, where $21.5 \%$ harbored two mutations (Table 4 ) and $12.9 \%$ harbored three mutations (Table 5). Of the 70 samples with mutations, 63 (90.0\%) had a mutation in APC, KRAS, and/or TP53; $17.1 \%$ had KRAS and TP53 mutations, $11.4 \%$ had a combination of KRAS and PIK3CA or KRAS and APC, 8.6\% with KRAS and FBXW7, and 5.7\% with APC and TP53. Not only were KRAS and TP53 mutations found at the highest frequency in our study, these mutations were also found most commonly in combination with mutations in other genes: $14 / 20(70.0 \%)$ of samples with two mutations had a KRAS mutation, and 9/20 (45.0\%) harbored a TP53 mutation. Of the double mutants, $25 \%$ were a combination of KRAS and TP53, and 25\% were KRAS and APC. Interestingly, all but one sample $(91.7 \%)$ with three mutations had at least one KRAS mutation, and $50 \%$ of these samples contained at least one FBXW7 mutation. Three samples $(25.0 \%)$ contained a combination of KRAS, TP53, and PIK3CA.

A previous study on gene mutations in European CRC patients found the most common combination mutations to be TP53 and APC, whereas mutations in TP53 co-occurring with KRAS were rare [25]; conversely, we found TP53 and APC combination mutations in only $4.3 \%$ of our samples and TP53 plus KRAS combination mutations in $12.9 \%$ of CRCs. Another study with Taiwanese CRC samples found the most common combination mutations to occur between APC and KRAS [48], whereas we identified that combination in only $8.6 \%$ of our samples. Taken together, these results indicate the wide variability in genetic mutations found in CRCs from different populations and underscores the need to further evaluate CRCs for common mutation patterns.

\section{Conclusion and future directions}

In the present study, we used Ion Torrent Ampliseq Cancer Panel to sequence 737 loci from 45 cancer-related genes, mainly oncogenes and tumor suppressor genes, in 93 human CRC samples. We identified frequent mutations in APC, FBXW7, KRAS, PIK3CA, and TP53 (Table 2), and the mutation profile was consistent with prior knowledge of genetic alterations in colorectal cancer [49]. In comparison with traditional Sanger sequencing and other Next Generation Sequencing (NGS) methods which utilize a 4-color optical detection system, the Ion Torrent sequencing platform has a major advantage in its Post-Light sequencing technology, which reduces both assay cost and complexity. This technology allows Ion Torrent 
Citation: Cai ZX, Tang XD, Gao HL, Tang C, Nandakumar V, et al. (2014) APC, FBXW7, KRAS, PIK3CA, and TP53 Gene Mutations in Human Colorectal Cancer Tumors Frequently Detected by Next-Generation DNA Sequencing. J Mol Genet Med 8: 145. doi: 10.4172/1747-0862.1000145

Page 10 of 11

sequencing to produce reliable results at a relatively faster rate and reduced sequencing costs per base [50].

In developed countries, colorectal cancer is the third most common malignancy and the second most frequent cause of cancer-related death. Additionally, prognosis is poor for patients with this disease and the overall 5-year survival rate is only $6.6 \%-11.9 \%$ [51], indicating that the traditional treatments of surgery, radiotherapy, and generalized chemotherapy are less than optimal. Hence, treatment of CRC has been quite a challenge for clinicians, and targeting specific molecular pathways and gene mutations has become a critical step in improving treatment options and increasing patient survival. Therefore, identifying and understanding the mutation profile of individual cancer genomes may provide valuable information on targeted treatments and potential routes for treatment.

A number of targeted agents have been developed, including EGFR, VEGF, and multi-kinase inhibitors, which in combination with chemotherapy have demonstrated improved outcome in metastatic colorectal cancer patients [52,53]. In addition to developing targeted agents to specific mutations, it is also important to understand their molecular action thoroughly in order to avoid any side- and countereffects. For example, patients harboring KRAS mutations are resistant to treatment with anti-EGFR monoclonal antibodies. Consistently, recent clinical evidences from phase II and phase III trials of Panitumumab and Cetuximab showed that patients with KRAS mutations in their tumors did not respond appropriately to the targeted drugs [54]. Currently KRAS mutation testing is routinely performed to prevent patients' exposure to unnecessary drug toxicities in situations where anti-EGFR monoclonal antibodies would not be beneficial. However, there are currently no specific treatment options for patients harboring KRAS mutations, and as KRAS is the most frequently mutated gene in CRCs, these patients are in critical need for alternative target therapies.

Accumulating evidence indicates that effective treatment for the majority of most malignancies requires more than the administration of a single agent but a combination of multiple therapies [55]. By identifying mutation combinations in individual cancers, such as in our study, directed treatments with a combination of targeted agents against the detected mutations may prove to have greater benefits for cancer patients. Ion Torrent sequencing may help provide clinicians a rapid, affordable, and feasible way to reliably identify mutations in CRCs to further improve patient treatments and outcomes in the near future.

\section{Acknowledgements}

We would like to thank Rong Shi at the Wu Jieping Foundation, Dr. Haibo Wang, Zhi Yu, Ying Li, and other members of San Valley Biotechnology Inc. Beijing for their assistance in sample and data collection. We would also like to thank the staff at the Beijing Military Hospital for their generous support for DNA sequencing and data collection. This research was supported by the grants from the $\mathrm{Wu}$ Jieping Foundation, the National Natural Science Foundation of China, No. 81273919, The natural science foundation of Liaoning Province, No. 2012225020 and the National Basic Research Program of China (973 Program), No. 2013CB531703, and the National Institute of Health (R01 CA90427 \& R01 AI084811 to SY Chen).

\section{References}

1. Ferlay J, Soerjomataram I, Ervik M, Dikshit R, Eser S, et al. (2013) GLOBOCAN 2012 v1.0, Cancer Incidence and Mortality Worldwide: IARC Cancer Base No. 11, International Agency for Research on Cancer.

2. Bray F, Ren JS, Masuyer E, Ferlay J (2013) Global estimates of cancer prevalence for 27 sites in the adult population in 2008. Int J Cancer 132: 1133-1145.

3. Cunningham D, Atkin W, Lenz HJ, Lynch HT, Minsky B, et al. (2010) Colorectal cancer. Lancet 375: 1030-1047.

4. He J, Efron JE (2011) Screening for colorectal cancer. Adv Surg 45: 31-44.

5. de la Chapelle A (2004) Genetic predisposition to colorectal cancer. Nat Rev Cancer 4: 769-780.

6. Watson AJ, Collins PD (2011) Colon cancer: a civilization disorder. Dig Dis 29: 222-228.

7. Key TJ, Allen NE, Spencer EA, Travis RC (2002) The effect of diet on risk of cancer. Lancet 360: 861-868.

8. Triantafillidis JK, Nasioulas G, Kosmidis PA (2009) Colorectal cancer and inflammatory bowel disease: epidemiology, risk factors, mechanisms of carcinogenesis and prevention strategies. Anticancer Res 29: 2727-2737.

9. Fishel R, Lescoe MK, Rao MR, Copeland NG, Jenkins NA, et al. (1993) The human mutator gene homolog MSH2 and its association with hereditary nonpolyposis colon cancer. Cell 75: 1027-1038.

10. Silvestri A, Pin E, Huijbers A, Pellicani R, Parasido EM, et al. (2013) Individualized therapy for metastatic colorectal cancer. J Intern Med 274: $1-24$.

11. Triantafillidis JK, Nasioulas G, Kosmidis PA (2009) Colorectal cancer and inflammatory bowel disease: epidemiology, risk factors, mechanisms of carcinogenesis and prevention strategies. Anticancer Res 29: 2727-2737.

12. Vogelstein B, Papadopoulos N, Velculescu VE, Zhou S, Diaz LA Jr, et al. (2013) Cancer genome landscapes. Science 339: 1546-1558.

13. Schnekenburger M, Diederich M (2012) Epigenetics Offer New Horizons for Colorectal Cancer Prevention. Curr Colorectal Cancer Rep 8: 66-81.

14. Lee AJ, Endesfelder D, Rowan AJ, Walther A, Birkbak NJ, et al. (2011) Chromosomal instability confers intrinsic multidrug resistance. Cancer Res 71: 1858-1870.

15. Hadd AG, Houghton J, Choudhary A, Sah S, Chen L, et al. (2013) Targeted, high-depth, next-generation sequencing of cancer genes in formalin-fixed, paraffin-embedded and fine-needle aspiration tumor specimens. J Mol Diagn 15: 234-247.

16. Bai X, Zhang E, Ye H, Nandakumar V, Wang Z, et al. (2014) PIK3CA and TP53 gene mutations in human breast cancer tumors frequently detected by ion torrent DNA sequencing. PLoS One 9: e99306.

17. Cai X, Sheng J, Tang C, Nandakumar V, Ye H, et al. (2014) Frequent mutations in EGFR, KRAS and TP53 genes in human lung cancer tumors detected by ion torrent DNA sequencing. PLoS One 9: e95228.

18. 1000 Genomes Project Consortium, Abecasis GR, Altshuler D, Auton A, Brooks LD, et al. (2010) A map of human genome variation from population-scale sequencing. Nature 467: 1061-1073.

19. Server EV (2013) NHLBI Go Exome Sequencing Project (ESP). Seattle, WA.

20. Bamford S, Dawson E, Forbes S, Clements J, Pettett R, et al. (2004) The COSMIC (Catalogue of Somatic Mutations in Cancer) database and website. Br J Cancer 91: 355-358.

21. Forbes SA, Bindal N, Bamford S, Cole C, Kok CY, et al. (2011) COSMIC: mining complete cancer genomes in the Catalogue of Somatic Mutations in Cancer. Nucleic Acids Res 39: D945-950.

22. Horii A, Nakatsuru S, Ichii S, Nagase H, Nakamura Y (1993) Multiple forms of the APC gene transcripts and their tissue-specific expression. Hum Mol Genet 2: 283-287.

23. Thliveris A, Albertsen H, Tuohy T, Carlson M, Groden J, et al. (1996) Long-range physical map and deletion characterization of the $1100-\mathrm{kb}$ 
Citation: Cai ZX, Tang XD, Gao HL, Tang C, Nandakumar V, et al. (2014) APC, FBXW7, KRAS, PIK3CA, and TP53 Gene Mutations in Human Colorectal Cancer Tumors Frequently Detected by Next-Generation DNA Sequencing. J Mol Genet Med 8: 145. doi: $10.4172 / 1747-0862.1000145$

Page 11 of 11

NotI restriction fragment harboring the APC gene. Genomics 34: 268-270.

24. Béroud C, Soussi T (1996) APC gene: database of germline and somatic mutations in human tumors and cell lines. Nucleic Acids Res 24: 121-124.

25. Smith G, Carey FA, Beattie J, Wilkie MJ, Lightfoot TJ, et al. (2002) Mutations in APC, Kirsten-ras, and p53--alternative genetic pathways to colorectal cancer. Proc Natl Acad Sci USA 99: 9433-9438.

26. Galiatsatos P, Foulkes WD (2006) Familial adenomatous polyposis. Am J Gastroenterol 101: 385-398.

27. Grim JE (2014) Fbxw7 hotspot mutations and human colon cancer: mechanistic insights from new mouse models. Gut 63: 707-709.

28. Orlicky S, Tang X, Willems A, Tyers M, Sicheri F (2003) Structural basis for phosphodependent substrate selection and orientation by the SCFCdc4 ubiquitin ligase. Cell 112: 243-256.

29. Hao B, Oehlmann S, Sowa ME, Harper JW, Pavletich NP (2007) Structure of a Fbw7-Skp1-cyclin E complex: multisite-phosphorylated substrate recognition by SCF ubiquitin ligases. Mol Cell 26: 131-143.

30. Akhoondi S, Sun D, von der Lehr N, Apostolidou S, Klotz K, et al. (2007) FBXW7/hCDC4 is a general tumor suppressor in human cancer. Cancer Res 67: 9006-9012.

31. Welcker M, Clurman BE (2008) FBW7 ubiquitin ligase: a tumour suppressor at the crossroads of cell division, growth and differentiation. Nat Rev Cancer 8: 83-93.

32. Akhoondi S, Sun D, von der Lehr N, Apostolidou S, Klotz K, et al. (2007) FBXW7/hCDC4 is a general tumor suppressor in human cancer. Cancer Res 67: 9006-9012.

33. Zoratto F, Rossi L, Verrico M, Papa A, Basso E, et al. (2014) Focus on genetic and epigenetic events of colorectal cancer pathogenesis: implications for molecular diagnosis. Tumour Biol 35: 6195-6206.

34. Bazan V, Migliavacca M, Zanna I, Tubiolo C, Grassi N, et al. (2002) Specific codon $13 \mathrm{~K}$-ras mutations are predictive of clinical outcome in colorectal cancer patients, whereas codon $12 \mathrm{~K}$-ras mutations are associated with mucinous histotype. Ann Oncol 13: 1438-1446.

35. Edkins S, O'Meara S, Parker A, Stevens C, Reis M, et al. (2006) Recurrent KRAS codon 146 mutations in human colorectal cancer. Cancer Biol Ther 5: 928-932.

36. Janakiraman M, Vakiani E, Zeng Z, Pratilas CA, Taylor BS, et al. (2010) Genomic and biological characterization of exon 4 KRAS mutations in human cancer. Cancer Res 70: 5901-5911.

37. Vogelstein B, Kinzler KW (2004) Cancer genes and the pathways they control. Nat Med 10: 789-799.

38. Stintzing S, Lenz HJ (2013) A small cog in a big wheel: PIK3CA mutations in colorectal cancer. J Natl Cancer Inst 105: 1775-1776.

39. Vogt PK, Kang S, Elsliger MA, Gymnopoulos M (2007) Cancer-specific mutations in phosphatidylinositol 3-kinase. Trends Biochem Sci 32: 342-349.
40. Bader AG, Kang S, Vogt PK (2006) Cancer-specific mutations in PIK3CA are oncogenic in vivo. Proc Natl Acad Sci U S A 103: 1475-1479.

41. Kang S, Bader AG, Zhao L, Vogt PK (2005) Mutated PI 3-kinases: cancer targets on a silver platter. Cell Cycle 4: 578-581.

42. Zhao L, Vogt PK (2008) Class I PI3K in oncogenic cellular transformation. Oncogene 27: 5486-5496.

43. Pfeifer GP, Besaratinia A (2009) Mutational spectra of human cancer. Hum Genet 125: 493-506.

44. Baker SJ, Preisinger AC, Jessup JM, Paraskeva C, Markowitz S, et al. (1990) p53 gene mutations occur in combination with $17 \mathrm{p}$ allelic deletions as late events in colorectal tumorigenesis. Cancer Res 50: 7717-7722.

45. Ory K, Legros Y, Auguin C, Soussi T (1994) Analysis of the most representative tumour-derived p53 mutants reveals that changes in protein conformation are not correlated with loss of transactivation or inhibition of cell proliferation. EMBO J 13: 3496-3504.

46. Sigal A, Rotter V (2000) Oncogenic mutations of the p53 tumor suppressor: the demons of the guardian of the genome. Cancer Res 60: 6788-6793.

47. Petitjean A, Achatz MI, Borresen-Dale AL, Hainaut P, Olivier M (2007) TP53 mutations in human cancers: functional selection and impact on cancer prognosis and outcomes. Oncogene 26: 2157-2165.

48. Wang JY, Hsieh JS, Lu CY, Yu FJ, Wu JY, et al. (2007) The differentially mutational spectra of the APC, K-ras, and p53 genes in sporadic colorectal cancers from Taiwanese patients. Hepatogastroenterology 54: 2259-2265.

49. Fearon ER (2011) Molecular genetics of colorectal cancer. Annu Rev Pathol 6: 479-507.

50. Roukos DH (2011) Trastuzumab and beyond: sequencing cancer genomes and predicting molecular networks. Pharmacogenomics J 11: 81-92.

51. Broaddus E, Topham A, Singh AD (2009) Survival with retinoblastoma in the USA: 1975-2004. Br J Ophthalmol 93: 24-27.

52. Van Cutsem E, Köhne CH, Hitre E, Zaluski J, Chang Chien CR, et al. (2009) Cetuximab and chemotherapy as initial treatment for metastatic colorectal cancer. N Engl J Med 360: 1408-1417.

53. Douillard JY, Siena S, Cassidy J, Tabernero J, Burkes R, et al. (2010) Randomized, phase III trial of panitumumab with infusional fluorouracil, leucovorin, and oxaliplatin (FOLFOX4) versus FOLFOX4 alone as firstline treatment in patients with previously untreated metastatic colorectal cancer: the PRIME study. J Clin Oncol 28: 4697-4705.

54. Lièvre A, Bachet JB, Le Corre D, Boige V, Landi B, et al. (2006) KRAS mutation status is predictive of response to cetuximab therapy in colorectal cancer. Cancer Res 66: 3992-3995.

55. Kahn M (2014) Can we safely target the WNT pathway? Nat Rev Drug Discov 13: 513-532. 